\title{
The Study of Architecture on Vehicle Information System
}

\author{
Pengfei Liu ${ }^{1, a}$, Yong Wang ${ }^{2, b}$, Pengfei Yin ${ }^{2, b}$ \\ ${ }^{1}$ College of Computer Science and Technology, Beijing University of Technology, Beijing, 100124, \\ China \\ aemail: liupengfeimail@163.com, bemail:yinpengfeimail@163.com
}

\section{Keywords: Vehicle Information System; MVC; Architecture}

\begin{abstract}
Vehicle Information System is one of the important components of the modern automobile electron. Because of the development of the science and technology and the huge market demands, In-vehicle electronic equipment which was the single in-vehicle navigation equipment at the very beginning is developing rapidly into a multi-functional Vehicle Information System. In order to obtain a good development environment, an excellent vehicle information system Architecture is indispensable for an enterprise. The paper will give an analysis of the MVC architecture for the vehicle information system.
\end{abstract}

\section{Introduction}

With the continuous development of science and technology, the function of the vehicle information system also has improved step by step. Most of the previous vehicle information equipment is made up of separate parts or simple modules[1]. System structure is lack of unified design, which leads to the overall system performance is poor, low configurability, function is simple. Using on-board computer as the platform, the vehicle communication, navigation, audio-visual entertainment, network control integration for the integration of vehicle information system is development trend of domestic auto electronics market[2]. This system can acquire and apply all kinds of information through data collection, information processing, and the connection of all kinds of information exchanging center and the net with the support of the data communication web. Besides, it also has the function of intelligence navigation, vehicle controlling, message safety defending and the multi-entertainment.

This paper Using MVC three layer c/s architecture, Implementation of special vehicle on-board information system architecture research. In the aspect of communication, Using master-slave communication architecture. Realizing all kinds of data monitoring such as running status information, environmental awareness information, location information, vehicle information communication, information of controlling of the vehicle and auxiliary driving and other kinds of information.

\section{Architecture of the Vehicle information System}

Vehicle information systems is a application software systems that run on the hardware platform and operating system platforms, divided into the following several levels:

Data Layer: the main sources of data is vehicle operation information, context-aware information and external data, such as audio and video etc, respectively through CAN bus, USB and other ways to collect them to database[3][4].

Data Storage Layer: Including running state of the vehicle information database, context-aware information database[5].

Human Interface Layer: First developed for different vehicle information corresponding to the content management panel, respectively, operating status information management panel, context-aware information management panel, driver assistance systems management panel and navigation information management panels etc; Secondly, different panels having a unified interface, in the form of software components exist, to facilitate integration in a unified framework; 
Finally, in the other car information, in the human-computer interaction interface layer, only the content of the need to develop obey uniform interface panel can be integrated into the integrated framework, easy to extend[6][7].

In addition, the vehicle information data can be compared to other vehicle information system and the vehicle center of cloud, interacting through a communication network.

The vehicle information system architecture Figure 1 is below:

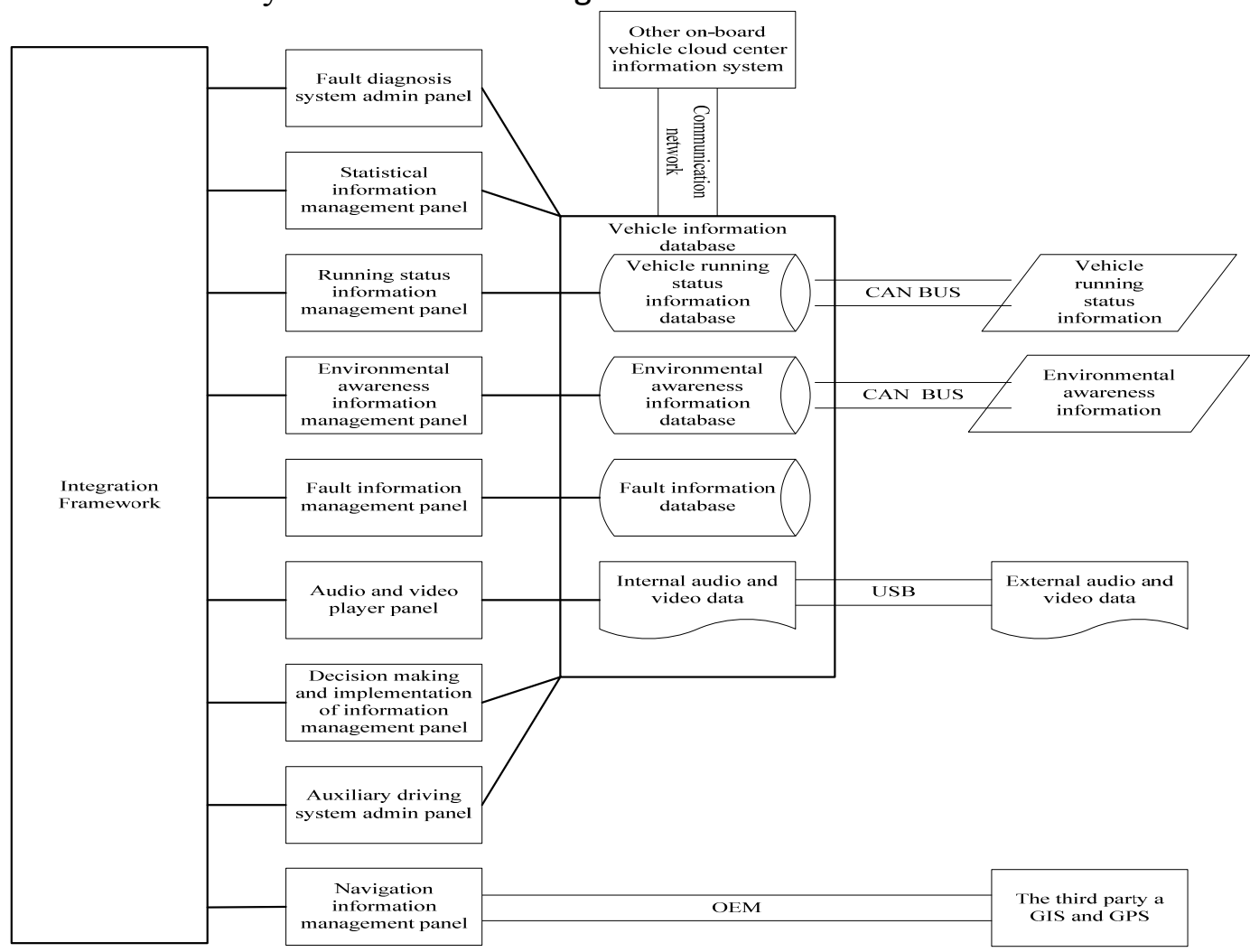

Fig 1 Vehicle information system architecture

\section{Functional Modules of the Vehicle information System}

Due to the special security requirements of special vehicles, lead to the complexity of the special vehicle on-board information system[8]. On one hand, vehicle systems need to have all civilian cars on-board system function, such as car networking applications in the future. On the other hand, special vehicle on-board system need special technology to guarantee its safety[9][10]. So, we need to split the overall architecture of special vehicle information system into four computers: Controlling the running machine, Information management machine, Security network communication machine, Public network communication machine. Running controlling machine, information management and security network communication machine connected through local area network operation controlling, information management and security network communication machine connected through the LAN.

The functional module Figure 2 is as following: 


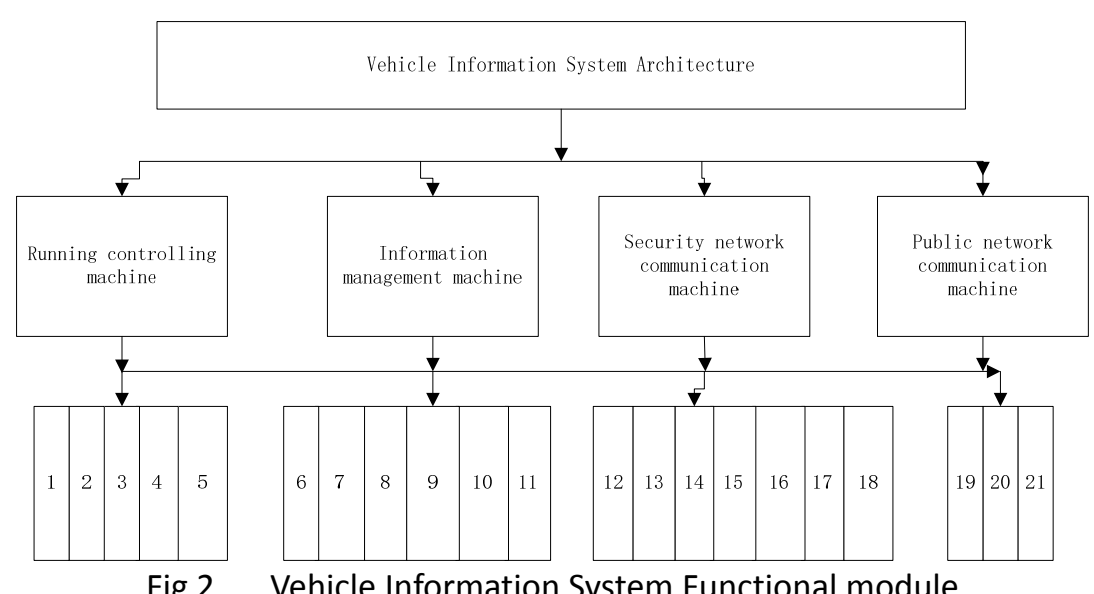

Fig 2 Vehicle Information System Functional module

Notes: (1)communication (2)monitoring awareness(5)help(6)communication(7)monitoring (10)Information querying (10)auxiliary driving (13)help (14)monitoring (15) environmental awareness (16)position navigation (17)cooperative engagement (18)remote operations (19)internet searching (20)Internet data querying (21)communication with other vehicle in the Internet. (3)auxiliary driving (8)Environmental (4)environmental awareness $(9)$ help

\section{Controlling the running machine}

Running controlling machine as the core part of the original vehicle information system, under the condition of trying to retain the original vehicle information system, to increase LAN networking module for running controlling machine, making its can be connected to the on-board LAN, and communicating. The display mode of running controlling machine information can still retain the original dashboard, increase the display screen mode of LCD touching. Its main functions for the vehicle operation information acquisition and vehicle controlling.

\section{Information management machine}

Information management machine provides all kinds of information about the vehicle management function, through the local area network and operation control machine, it can obtain the vehicle related all kinds of information; on the other hand, Information management machine through local area network and security of network communication machine, it can obtain information of other vehicles. On the one hand, information management machine information provides all kinds of vehicles related storage and management function; on the other hand, provide information based on the vehicle and grew out of a variety of applications.

\section{Security network communication machine}

Security network communication machine is responsible for vehicles' security network connectivity, including through the network security, such as digital radio, to communicate with other vehicles; Also through the network security, such as military private network, and military vehicles cloud center, such as remote command center, the communication function, various remote access to military vehicles cloud center applications.

\section{Public network communication machine}

Public network communication machine is responsible for vehicles and public network connection. It is important to note, it is physical isolation to the other vehicle in the internal network . 


\section{Conclusion}

The successful implementation of the architecture of the vehicle information system can improve the forces survival and combat effectiveness. With the architecture, we successfully build a satisfied vehicle information system to our country. In addition, it also provides a reference for the construction of other vehicle information system.

\section{References}

[1] Lars B, Frerdriksson. CAN for critical embedded automotive networks[J].IEEE Micro,2002,(04):28-35.doi:10.1109/MM.2002.1028473.

[2] Analog Device Inc. EE-256 using the ADSP-BF561 blackfin(R)processor as a TFT-LCD controller[OL].http://www,analog,com/en/index,html,2010.

[3] Analog Device Inc. ADSP-BF537 blackfin(R)processor hardware reference[OL].http://www.analog.com/en/index,html,2010.

[4] Ran Li,Junfeng Wu,Haiying Wang. Design method of CAN BUS network communication structure for electric vehicle[A].Ulsan,Korea: IEEE,2010.

[5] Chen I-An,Cheng Chang-Hsin,Jheng Hong-Yuan. An error-correction scheme with Reed-Solomon codec for CAN bus transmission[A].Chiang Ma,Thailand: IEEE,2011.

[6] Xu Yang,Wilfred W. Recker. Modeling Dynamic Vehicle Navigation in a Self-Organizing, Peer-to-Peer, Distributed Traffic Information System[J].Journal of Intelligent Transportation Systems, 2006, Vol.10

[7] Han-Chun, Yeh,Su-YingChang, Yuan-ShengChu,Chien-WeiChen,Chi-Sheng Shih.Vehicle Information Systems Integration Framework[J]. J. Inf. Sci. Eng., 2007, Vol.23

[8] Huang Zuowei, Zhou Ming, Zhang Ximei. Design and Realization of Embedded Vehicle Termi nal Based on GPS / GPRS. Computer Measurement \& control, Magn. China, vol. 17, no. 11, pp. 22 05-2208, 2009.

[9]Ronald Ecker, Verena Broy, Katja Hertzschuch, Andreas Butz . Visual cues supporting direct touch gesture interaction with in-vehicle information systemsRonald Ecker, Verena Broy, Katja Hertzschuch, Andreas Butz[J]. MobileHCI '09: Proceedings of the 11th International Conference on Human-Computer Interaction with Mobile Devices and Services September 2009.

[10]An extended keystroke level model (KLM) for predicting the visual demand of in-vehicle information systems[J]. CHI '07: Proceedings of the SIGCHI Conference on Human Factors in Computing Systems April 2007 